\title{
Expiratory positive airway pressure (EPAP) nasal device therapy: a welcome addition to obstructive sleep apnea syndrome therapy
}

\author{
Sophia E. Schiza • Charalampos Mermigkis • \\ Izolde Bouloukaki
}

Received: 28 September 2014 / Revised: 7 October 2014 / Accepted: 10 October 2014 / Published online: 7 April 2015

(C) Springer-Verlag Berlin Heidelberg 2015

Obstructive sleep apnea-hypopnea syndrome (OSAHS) is characterized by intermittent partial or complete occlusion of the upper airway, affecting 5 to $10 \%$ of middle-aged adults and up to $20 \%$ of adults over 65 years of age [1]. Treatments for patients with OSAHS include positive airway pressure (PAP) therapy, oral appliances, various surgeries that modify the upper airway, and/or weight loss. However, it is well documented that there is lack of acceptance or suboptimal adherence to these available medical therapies $[2,3]$. Therefore, given the high prevalence of OSAHS, new effective treatment options would be welcomed.

Alternative management approaches for OSAHS, less intrusive and simpler to use, have emerged. One of these alternatives is expiratory positive airway pressure (EPAP) nasal device, a small, lightweight, and quiet device that does not require an external power source. There are several mechanisms by which nasal EPAP devices positively impact OSAHS. These disposable adhesive devices are placed over the nostrils and increase resistance to flow during exhalation while not adversely affecting flow during inhalation. This results in positive pressure application throughout exhalation and in an upper airway being more resistant to collapse on subsequent inspiration [4].

In their study reported in this Journal, Dr. Hao and colleagues performed a systematic review about the effectiveness of EPAP treatment as an alternative therapy for OSAHS. Based on this review, the effectiveness of nasal EPAP has been validated through published clinical trials which demonstrate statistically significant and clinically meaningful

S. E. Schiza $(\triangle) \cdot$ C. Mermigkis $\cdot$ I. Bouloukaki

Sleep Disorders Center, Department of Thoracic Medicine,

University General Hospital, Medical School of the University of

Crete, 71110 Heraklion, Crete, Greece

e-mail: schiza@med.uoc.gr reductions in the apnea-hypopnea index (AHI), oxygen desaturation, and daytime sleepiness, with concomitant improvements in quality of life and sleep quality. The greatest benefit was observed in those patients with more mild disease. Some children may also respond to nasal EPAP, but there is an individual variability in their response [5]. Adverse events associated with device use were judged to be mild to moderate and did not significantly impact adherence for patients demonstrating initial tolerability of EPAP. Median device use was excellent, with self-reported adherence to device treatment exceeding $88 \%$ in a large trial [4].

Although these results are encouraging, in practice, proper nightly application of the device is difficult for many patients even with proper education, probably resulting in suboptimal adherence. Furthermore, adherence data in all studies may be artificially high due to the relatively short duration of the studies, frequent interaction by study staff, and because the data were obtained by diary rather than by an objective method such as that typically employed to monitor PAP use. It is also possible that motivation may be higher in patients who have had prior difficulty using PAP or who seek participation in a clinical trial for a new sleep apnea treatment. Therefore, adherence data will need to be confirmed with additional objective demonstration of treatment adherence.

It is important to note that after acclimatization to nasal EPAP during the initial period, patients should undergo an assessment of effectiveness to ensure a satisfactory therapeutic benefit. The same methodology used for OSAHS diagnosis is suggested, including in-lab polysomnography (PSG) or portable recording to obtain objective results. Reportedly, one patient has developed complex sleep apnea with use of both nasal EPAP and PAP devices, more severe with the nasal EPAP device during the supine position, highlighting the importance of obtaining objective sleep data prior to longterm prescription of a nasal EPAP device for treatment of 
OSAHS [6]. In addition, the physician who elects to continue treatment for extended periods should monitor the patient's clinical progress and periodically re-evaluate the long-term usefulness of the device. Factors that should also be considered are the insurance coverage, requirements for special nasal pressure cannulas in the sleep laboratory to objectively document efficacy, and the inability to objectively document efficacy on an ongoing basis.

Despite the high self-reported adherence rates, there is a considerable heterogeneity in response to this device. As the authors of this review explain, the magnitude of improvement with nasal EPAP is generally less than what is typically reported for PAP; thus, many patients are not completely treated. Untreated OSAHS has been associated with several adverse outcomes, especially cardiovascular complications, which are dose dependent in the context of an increased $\mathrm{AHI}$ and the severity of oxygen desaturation. Data suggest that even a low AHI confers increased risk for adverse effects. However, one should keep in mind the difference between efficacy and effectiveness as it relates to the use of therapy for the treatment of OSAHS. These terms are not synonymous. While normalization of AHI is a logical and well-accepted efficacy standard for treatment of OSAHS, a high level of adherence with treatment is needed for a similarly high degree of treatment effectiveness. For example, PAP is well established as being very efficacious, reducing the AHI to less than $5 / \mathrm{h}$, but its low adherence rates limit its effectiveness, meaning that it cannot eliminate all respiratory events. This distinction is, unfortunately, not always understood by practitioners and is of significant clinical importance because even the best therapies do not work if they are not used. In other words, some less efficacious treatment with high adherence may be a comparable or better alternative than a highly efficacious treatment with poor adherence. Therefore, as there is a high adherence rate, the effectiveness of nasal EPAP compares favorably to other treatment modalities.

The question that arises is what is the patient or the particular phenotype that may be ideal for EPAP therapy. Individual differences in the mechanisms contributing to sleep apnea such as ventilatory control instability, airway collapsibility, and arousal threshold probably play a role. Of note, PAP treatment has the potential to "attack" all possible phenotypes. At this time, there are no indications to predict which patients will be most effectively treated with nasal EPAP based on specific patient characteristics. Based on the present results, patients with mild to moderate OSAHS without significant comorbidities seem to be the best candidates for EPAP treatment and patients with severe OSAHS should first consider PAP therapy, which is the gold standard treatment. Moreover, nasal EPAP may be considered for patients who have rejected or are non-compliant with prescribed PAP, or PAP compliant patients who are looking for alternatives to their current therapy or for travel. It is appropriate to question why EPAP is efficacious in some patients and not in others. A pilot study by Patel and colleagues was not able to establish predictors of success but showed that subjects with positional OSAHS may be more likely to respond to this therapy [7], an observation that needs to be confirmed with a larger study.

In clinical practice, the clinician frequently has to decide between a therapy that has a better success rate (such as PAP therapy) and other available treatments that have relatively lower response rates but are more likely to be used by the patient. In this context, it appears that the EPAP device may be an alternative treatment for patients with mild OSAHS or those who are intolerant of PAP therapy. If used, testing is required to assess effectiveness of therapy. Potential responder phenotypes are insufficiently defined, and further research is required to identify the ideal candidates for this new therapeutic option of OSAHS.

\section{References}

1. Jordan AS, McSharry DG, Malhotra A (2014) Adult obstructive sleep apnoea. Lancet 383:736-747

2. Weaver TE, Grunstein RR (2008) Adherence to continuous positive airway pressure therapy: the challenge to effective treatment. Proc Am Thorac Soc 15:173-178

3. Wolkove N, Baltzan M, Kamel H, Dabrusin R, Palayew M (2008) Long-term compliance with continuous positive airway pressure in patients with obstructive sleep apnea. Can Respir J 15:365-369

4. Berry RB, Kryger MH, Massie CA (2011) A novel nasal expiratory positive airway pressure (EPAP) device for the treatment of obstructive sleep apnea: a randomized controlled trial. Sleep 34:479-485

5. Kureshi SA, Gallagher PR, McDonough JM, Cornaglia MA, Maggs J, Samuel J, Traylor J, Marcus CL (2014) Pilot study of nasal expiratory positive airway pressure devices for the treatment of childhood obstructive sleep apnea syndrome. J Clin Sleep Med 10:663-669

6. Chopra A, Das P, Ramar K, Staats B, St Louis EK (2014) Complex sleep apnea associated with use of nasal expiratory positive airway (nEPAP) device. J Clin Sleep Med 10:577-579

7. Patel AV, Hwang D, Masdeu MJ, Chen GM, Rapoport DM, Ayappa I (2011) Predictors of response to a nasal expiratory resistor device and its potential mechanisms of action for treatment of obstructive sleep apnea. J Clin Sleep Med 7:13-22 